\title{
Hydrophobic copper nanowires for enhancing condensation heat transfer
}

Rongfu Wen ${ }^{1,2, *}$, Qian $\mathrm{Li}^{1, *}$, Jiafeng $\mathrm{Wu}^{1,3, *}$, Gensheng $\mathrm{Wu}^{1,4}$, Wei Wang ${ }^{1,5}$, Yunfei Chen ${ }^{6}$, Xuehu Ma ${ }^{2}$, Dongliang Zhao ${ }^{1}$, and Ronggui Yang ${ }^{1,7, \#}$

${ }^{1}$ Department of Mechanical Engineering, University of Colorado, Boulder, CO 80309-0427, USA

${ }^{2}$ Liaoning Key Laboratory of Clean Utilization of Chemical Resources, Institute of Chemical Engineering, Dalian University of Technology, Dalian 116024, P. R. China

${ }^{3}$ Key Laboratory of Energy Thermal Conversion and Control of Ministry of Education, School of Energy and Environment, Southeast University, Nanjing 210096, P. R. China

${ }^{4}$ School of Mechanical and Electronic Engineering, Nanjing Forestry University, Nanjing 210037, P. R. China

${ }^{5}$ Advanced Li-ion Batteries Engineering Lab, Ningbo Institute of Material Technology and Engineering, Chinese Academy of Sciences, Ningbo 315201, P. R. China

${ }^{6}$ Jiangsu Key Laboratory for Design \& Manufacture of Micro/Nano Biomedical Instruments and School of Mechanical Engineering, Southeast University, Nanjing 210096, P. R. China

${ }^{7}$ Materials Science and Engineering Program, University of Colorado, Boulder, CO 80309-0596, USA

* These authors contribute equally.

${ }^{\#}$ Corresponding author: Ronggui.Yang@Colorado.Edu 


\begin{abstract}
Rapid droplet removal by regulating surface topology and wettability has been exploited in nature and it is of great importance for a broad range of technological applications including water desalination and harvesting, power generation, environmental control, and thermal management. Recently there have been tremendous efforts in developing nanostructured surfaces for wettability control and enhancing phase-change heat transfer. However, the tendency of condensed droplets to form as pinned state rather than mobile mode on the nanostructured surfaces is likely to limit the applicability of such functionalized surfaces for condensation heat transfer enhancement. Here, we demonstrate enhanced condensation heat transfer on a nanowired hydrophobic copper surface where molecular permeation of water vapor into the separations between nanowires is greatly decreased, rendering spatial control on droplet nucleation and wetting dynamics. We show experimentally and theoretically that this novel strategy allows to achieve a 100\% higher overall heat flux over a broadened surface subcooling range, up to $24 \mathrm{~K}$, due to highly efficient droplet jumping compared to the state-of-the-art hydrophobic surfaces. These findings reveal that the droplet behaviors and condensation modes can be regulated by spatially controlling the nucleation events on the nanostructured surfaces, which paves the way for the design of nanostructured surfaces for enhanced phase-change heat transfer.
\end{abstract}

Keywords: copper nanowires, enhanced condensation, heat transfer, spatial control, droplet nucleation, droplet dynamics. 


\section{Introduction}

Condensation is a ubiquitous phase-change phenomenon [1-3] and has been widely used in energy-intensive industrial applications including water desalination and harvesting $[4,5]$, power generation [6] and environmental control [7], and thermal management of electronic systems [810]. Condensation heat transfer performance is highly dependent on surface property and its enhancement promises considerable savings in energy and resources [11-14]. For dropwise condensation on plain hydrophobic surfaces, the gravity-driven droplet removal helps refresh the condensing surface and allows 5-7 times better heat transfer performance when compared to the filmwise condensation [15]. There have been many efforts in promoting dropwise condensation heat transfer through the control of surface wettability [16-18]. Recent studies on nanostructured superhydrophobic surfaces demonstrated that small condensed droplets $(<100 \mu \mathrm{m})$ can undergo coalescence-induced droplet jumping which is independent of gravity [19-23], offering a new route to further enhance dropwise condensation heat transfer. Condensation on such functionalized surfaces undergoes four distinct stages including initial nucleation, individual droplet growth, coalescence of droplets, and droplet departure. Understanding the dynamic growth process of condensed droplets from a few nanometers in the nucleation stage to hundreds of micrometers in the shedding stage is the key to developing novel functionalized surfaces for highly efficient droplet jumping and enhanced heat transfer. Careful control of surface structure $[24,25]$, nucleation density [21, 26], droplet morphology [27], and departure dynamics [28, 29] is of great need for the design of nanostructured surfaces for enhancing condensation heat transfer.

Despite the intensive efforts on optimizing micro/nano-structured surfaces for manipulating droplet dynamics [25, 29-34], only recently enhanced condensation heat transfer utilizing the 
droplet jumping mechanism has been experimentally demonstrated on nanostructured superhydrophobic surfaces compared to conventional dropwise condensation on the plain hydrophobic surface, but at very small surface subcooling $(\Delta T<5 \mathrm{~K})[26,35]$. At larger surface subcooling, uncontrolled heterogeneous nucleation leads to a flooding condensation mode, which limits the heat transfer enhancement and thus hindering practical applications of such functionalized micro/nanostructured surfaces. Since the free energy barrier for droplet formation is strongly governed by surface wettability, delicate surface partition with wettability contrast has also been explored to spatially control droplet nucleation $[4,36]$. However, such surfaces exploiting wettability contrast work only at very small heat flux [37], which inevitable limits its application in pure steam, especially for large surface subcooling.

Significantly different from the above-reviewed approaches that control droplet formation by manipulating surface wettability contrast, in this work we propose a new scheme to control droplet nucleation by exploring the spatial confinement effect, which takes advantage of the reduced molecular permeability of vapor into the separations between nanowires on the condensing surface. By regulating the density difference of vapor between inside and outside of the separations, droplet nucleation tends to occur at the top nanowires for larger supersaturation, which mitigates droplet formation in separations between nanowires. The novel hydrophobic nanowires allow to achieve a 100\% higher heat flux over a broadened subcooling range, up to 24 $\mathrm{K}$, due to the highly efficient droplet jumping, compared to state-of-the-art dropwise condensation.

\section{Experimental section}

\section{Preparation of condensing surfaces}


Due to the wide applications of copper in thermal systems, high purity copper block $(99.9 \%$ purity) was used to fabricate the test samples in this work. Each test sample was polished by 2000 grit sandpaper, cleaned in an ultrasonic bath with acetone for 10 minutes, and then rinsed with isopropyl alcohol, ethanol, and deionized (DI) water. The samples were then dipped into a 2.0 M hydrochloric acid solution for $10 \mathrm{~min}$ to remove the native oxide film on the surface, then triple-rinsed with DI water, and dried with clean nitrogen gas. For comparison, both plain and nanowired hydrophobic surface has been fabricated as the condensing surfaces on the block.

Copper nanowires on the surface were fabricated by a two-step porous anodic alumina (PAA) template-assisted electro-deposition method (Supplementary Section 1). The PAA template was first bonded onto the sample surface by electrodepositing at $-0.8 \mathrm{~V}$ for 15 minutes. During the deposition process, short nanorods $(\sim 1 \mu \mathrm{m})$ were formed to serve as the screws to connect the PAA and sample surface. In the second step, copper nanowires were fabricated by depositing $\mathrm{Cu}$ in a three-electrode electroplating cell with the same electrolyte as that in the first step. The length of copper nanowires was controlled by the electrodeposition time. Here, the nanowires with an average height (length) of $20 \mu \mathrm{m}$ and $30 \mu \mathrm{m}$ were fabricated by the electrodeposition for 150 and 240 minutes, respectively. After immersing in $1 \mathrm{M} \mathrm{NaOH}$ solution to dissolve the PAA templates and cleaning with DI water, copper nanowired surfaces were obtained.

Hydrophobic functionalization of both the plain and nanowired surfaces was obtained by immersing the samples into a hot $\left(70{ }^{\circ} \mathrm{C}\right)$ ethanol solution of $2.5 \mathrm{mM}$ n-octadecanethiol $(96 \% \mathrm{n}$ octadecyl mercaptan) for 60 minutes. While the self-assembled hydrophobic coating thickened the diameter of nanowires by $\sim 3 \mathrm{~nm}$ due to the deposition of $n$-octadecyl mercaptan, the general morphology of nanowired surfaces was left unchanged [38]. 


\section{Surface characterizations}

The field-emission scanning electron microscopy (FE-SEM, JEOL JSM-7401F) was employed to examine the morphologies of the plain and nanowired hydrophobic surfaces. The focused ion beam (FIB, FEI NOVA 660i) instrument was used to cut the sample for crosssectional morphology observation. The water contact angle of condensing surfaces was measured by using an optical imaging system. A $5 \mu 1$ water droplet was placed at different locations on the surface. The contact angles were measured and averaged over six measurements.

\section{Condensation experiment}

Condensation experiments were carried out under saturated conditions using a custom-made chamber where the condensing condition can be precisely controlled and adjusted in a board range (Supplementary Section 2). The condensing surface, $40 \mathrm{~mm}$ in diameter, was mounted vertically in the chamber. Prior to performing the condensation experiments, the test chamber was evacuated to a pressure $P<5 \pm 0.5 \mathrm{~Pa}$ and the water was vigorously boiled to eliminate noncondensable gases. Throughout the experiments, the chamber pressure and temperature were continuously monitored to ensure saturated conditions. The temperature of the condensing surface was independently controlled via a chilled water bath, and inlet and outlet of cooling water were measured to determine the condensation heat flux $(q)$. Here the surface subcooling $(\Delta T)$ is defined as the temperature difference between the water vapor temperature $\left(T_{\mathrm{v}}\right)$ at the working pressure $(60 \pm 0.5 \mathrm{kPa})$ and the temperature of condensing surface $\left(T_{\mathrm{w}}\right)$, which can be obtained by extrapolating the temperature distribution in the condensing block. All values of the temperature, pressure and flow rate were collected by the data acquisition to determine the uncertainty of heat flux, surface subcooling, and heat transfer coefficient (Supplementary Section 3). The dynamic behaviors of condensed droplets were observed and visualized by an optical 
microscope and a high speed camera (Photron FASTCAM SA4). The vapor pressure tested (60 $\mathrm{kPa}$ ) corresponds to steam saturation temperature of $86^{\circ} \mathrm{C}$, which is the common condition for multi-effect evaporation desalination, low temperature heat pump, and heat pipe [14, 39].

\section{Results and discussion}

Fig. 1a-b shows the scanning electron microscope (SEM) images of the closely packed copper nanowires on copper surface with an average diameter $(d)$ of 200-240 nm, a height $(h)$ of $20 \mu \mathrm{m}$, and an average separation $(l)$ of $100-140 \mathrm{~nm}$. The apparent contact angle of a $5 \mu \mathrm{L}$ water droplet on the surface is $139.2 \pm 3.5^{\circ}$ (inset of Fig. 1b). The length of nanowires is controlled by electro-deposition time while the separation between nanowires is controlled by the structure of PAA templates. Fig. 1c illustrates the density difference of vapor molecules due to the spatial confinement effect of high aspect ratio separations $(h / l=150-200)$ between nanowires, which prevent vapor molecules from entering easily into the interior of nanostructures. As a result, the hydrophobic nanowires mitigate droplet formation in the separations, which could lead to mobile droplets and thus enhanced condensation heat transfer over a wide range of surface subcooling.

To determine the overall condensation heat transfer performance, condensing surfaces were tested vertically in a custom-built condensation experimental chamber (Supplementary Section 2). Fig. 2a shows the measured heat flux (left) and heat transfer coefficient (right) as a function of surface subcooling. Comparing to dropwise condensation on a plain hydrophobic surface (prepared by similar hydrophobic coating process on a plain copper surface, apparent contact angle of water is $114.6 \pm 2.6^{\circ}$ ), significant enhancement on both heat flux and heat transfer coefficient are observed on the nanowired hydrophobic surface (20 $\mu \mathrm{m}$ long nanowires) over a wide range of surface subcooling, up to $28 \mathrm{~K}$. As expected, the heat flux of dropwise condensation on the plain hydrophobic surface increases monotonically with the increase of 
surface subcooling due to the increasing growth rate and high departure frequency of condensed droplets. However, the heat flux of nanowired hydrophobic surfaces is highly dependent on droplet wetting states and dynamic behaviors at various surface subcooling (see next paragraph for the discussion). The heat flux reaches its maximum as the surface subcooling increases to $\Delta T$ $=8 \mathrm{~K}$ and then slightly reduces as the surface subcooling is increased to $\Delta T=15 \mathrm{~K}$. With further increase of surface subcooling, the heat flux increases monotonically, following the similar trend as dropwise condensation on the plain hydrophobic surface. Similar enhancement on the heat flux and heat transfer coefficient are also observed on the nanowired hydrophobic surface with $30 \mu \mathrm{m}$ long nanowires over a wide range of surface subcooling, up to $24 \mathrm{~K}$ (Fig. 2a). Compared to the heat transfer performance of hydrophobic surface with $20 \mu \mathrm{m}$ long nanowires, both the heat flux and heat transfer coefficient of the condensing surface with $30 \mu \mathrm{m}$ long nanowires slightly decrease, which is due to the increased thermal resistance of nanowire layer. Most excitingly, the heat flux significantly exceeds the previously reported heat transfer threshold (80 $\mathrm{kW} / \mathrm{m}^{2}$ ) on the nanostructured superhydrophobic surfaces where the flooding phenomena happens when surface subcooling is larger than $5 \mathrm{~K}$ [26].

Fig. 2b-d shows typical optical visualization images of condensed droplets on the nanowired hydrophobic surface (20 $\mu \mathrm{m}$ long nanowires) at different surface subcooling (Supplementary Videos for droplet dynamics). Based on droplet behaviors, the heat transfer enhancement can be divided into three regimes (condensation modes) according to the surface subcooling: 1) jumping condensation where the droplet dynamic is independent of gravity at small surface subcooling $(\Delta T<8 \mathrm{~K}) ; 2)$ dropwise condensation where droplet removal depends strongly on gravity at large surface subcooling ( $\Delta T>15 \mathrm{~K})$; and 3) mixing condensation at the intermediate surface subcooling $(8 \mathrm{~K}<\Delta T<15 \mathrm{~K})$. When the surface subcooling is small $(\Delta T<$ 
$8 \mathrm{~K}$ ), droplet removal is mostly due to droplet jumping, where the best heat transfer performance with more than $100 \%$ enhancement over dropwise condensation on the plain hydrophobic surface for both heat flux and heat transfer coefficient is observed. As shown in Fig. 2b, the suspended states of droplets are favorable for coalescence-induced droplet jumping ( $\Delta T=1.2 \mathrm{~K}$ ). After reaching its maximum at the transition surface subcooling $(\Delta T=8 \mathrm{~K})$, the heat flux enters another regime where droplets tend to form as pinned state rather than mobile mode. As a result, most of merged droplets after coalescence still stay on the surface although the coalescenceinduced droplet jumping is also observed (Fig. 2c). With further increase of surface subcooling, condensed droplets completely transition into the pinned states where the droplet removal relies on gravity (Fig. 2d). The droplet wetting transition leads to the failure of nanowired hydrophobic surfaces and the heat transfer performance tends to follow the trend on the plain hydrophobic surface. In summary, we report here significantly enhanced condensation heat transfer on nanowired hydrophobic surfaces over a wide subcooling range up to $24 \mathrm{~K}$ while the past works have been limited to $5 \mathrm{~K}[9,26]$.

To understand the heat transfer enhancement mechanisms of nanowired hydrophobic surfaces, we performed quantitative analysis on the droplet behaviors using the in-situ visualization built in our condensation heat transfer set-up (Supplementary Section 4). The droplets behave very differently on nanowired hydrophobic surfaces from those on the plain hydrophobic surfaces. Fig. 3a-b shows the typical growth process of a droplet from its formation to removal (droplet radius growing from zero to its maximum) under different surface subcooling. On the plain hydrophobic surface, the droplet growth rate increases up to $300 \%$ with the increase of surface subcooling from $6 \mathrm{~K}$ to $25 \mathrm{~K}$ (Fig. 3a), while the removal size of droplets remains about the same $(300-400 \mu \mathrm{m})$. Due to the wetting transition of condensed droplets on 
nanowired hydrophobic surfaces, however, drastic differences in the removal size and renewal frequency of droplets are observed at various surface subcooling, as shown in Fig. 3b. High renewal frequency and small removal size $(\sim 100 \mu \mathrm{m})$ of condensed droplets are achieved at the surface subcooling of $3 \mathrm{~K}$. With the increase of surface subcooling, droplet renewal frequency reduces and larger removal size $(\sim 600 \mu \mathrm{m})$ is observed. Fig. 3c shows the maximum droplet radius (removal radius) as function of surface subcooling. Due to the coalescence-induced droplet jumping, the maximum radius of droplets on the nanowired hydrophobic surface are smaller than $200 \mu \mathrm{m}$ at small surface subcooling $(\Delta T<5 \mathrm{~K})$, which is far smaller than those on the plain hydrophobic surface with a typical size of $400-450 \mu \mathrm{m}$. However, with the increase of surface subcooling ( $8 \mathrm{~K}<\Delta T<15 \mathrm{~K})$, droplet wetting begins to transition from mobile mode to pinned state, which leads to a rapid increase of maximum droplet radius. As the surface subcooling increases to $15 \mathrm{~K}$, droplets become completely pinned state which results in larger droplets on the nanowired hydrophobic surface than that on the plain hydrophobic surface. With the further increase of surface subcooling $(\Delta T>15 \mathrm{~K})$, the maximum droplet radius become almost constant $(\sim 600 \mu \mathrm{m})$ which relies on gravity for droplet removal. For the plain hydrophobic surface, the maximum droplet radius remains almost unchanged except a slight reduction under small surface subcooling $(\Delta T<8 \mathrm{~K})$, which is attributed to the same gravitydriven droplet removal mechanism.

Fig. 3d shows the renewal frequency of condensed droplets as a function of surface subcooling, which also exhibits remarkably different trends for the plain hydrophobic surface and nanowired hydrophobic surface. The renewal frequency of droplets on the plain hydrophobic surface increases monotonically with the increase of surface subcooling, which is attributed to the increased droplet growth rate. However, the renewal frequency of droplets on the nanowired 
hydrophobic surface decreases rapidly with the increase of surface subcooling from $5 \mathrm{~K}$ to $15 \mathrm{~K}$ and then increase again but slowly with the increase of surface subcooling when it is larger than $15 \mathrm{~K}$. Apparently, the renewal frequency of droplets on the nanowired hydrophobic surface is far higher than that for the plain hydrophobic surface at small surface subcooling $(\Delta T<5 \mathrm{~K})$, which is attributed to the coalescence-induced droplet jumping on the nanowired hydrophobic surface. At a large surface subcooling $(\Delta T>15 \mathrm{~K})$, condensed droplets stay on the surface as the pinned state, which results in a larger droplet adhesion compared to that on the plain hydrophobic surface. At large surface subcooling, droplet removal on nanowired hydrophobic surfaces still relies on gravity, but with a slightly lower frequency than that on the plain hydrophobic surface.

The visualization study of droplet behaviors can be directly used to estimate the condensation heat transfer performance. The condensation heat flux $q^{\prime}$ is related to the mean volume flux of condensate by $q^{\prime} \propto \rho_{\mathrm{l}} h_{\mathrm{fg}} v$, where $\rho_{\mathrm{l}}, h_{\mathrm{fg}}$ are the liquid density and the latent heat of phase change, and $v$ is the mean volume flux of condensate, which can be further written as $v \propto r_{\max } f_{\mathrm{s}}$. Fig. 3e shows the mean volume flux of condensate on the plain and nanowired hydrophobic surfaces. It can easily be seen that the trends for the mean volume flux of condensate obtained from the visualization study agrees well with the experimental heat transfer curves (Fig. 2a).

As discussed above, we achieve significant condensation heat transfer enhancement over a wide range of surface subcooling (up to $24 \mathrm{~K}$ ) on nanowired hydrophobic surfaces, in comparison with the previously reported enhanced condensation over a very small surface subcooling $(\Delta T<5 \mathrm{~K})$ by taking advantage of spatial confinement effect to mitigate droplet formation in the separations between nanowires. At large surface subcooling $(\Delta T>15 \mathrm{~K})$, 
however, due to the nucleation of low-density vapor molecules in the separations, the nanowired hydrophobic surface begins to fail and the heat transfer curve becomes similar to that on the plain hydrophobic surface. Here, we use the nucleation theory $[40,41]$ to explain the observation. Condensation begins with the formation of small clusters. When the size of the clusters exceeds a critical size that is determined by the interfacial and volumetric contributions to the Gibbs free energy, the clusters will grow further and form the macroscopic liquid phase. The Gibbs free energy barrier for droplet nucleation can be written as [36],

$$
\Delta G\left(r_{\mathrm{e}}\right)=\frac{\pi \sigma_{\mathrm{lv}} r_{\mathrm{e}}^{2}\left(2-3 \cos \theta+\cos ^{3} \theta\right)}{3}
$$

where $\sigma_{\mathrm{lv}}, \theta$, and $r_{\mathrm{e}}$ are the liquid-vapor surface tension, the intrinsic contact angle of water on hydrophobic coating, and the critical nucleation radius of droplets, respectively. Specifically, the critical nucleation radius can be given by the Kelvin's classical equation [42],

$$
r_{\mathrm{e}}=\frac{2 T_{\mathrm{v}} \sigma_{\mathrm{lv}}}{h_{\mathrm{fg}} \rho_{\mathrm{l}} \Delta T}
$$

According to equations (1) and (2), larger surface subcooling results in a smaller energy barrier for droplet nucleation whereas fewer vapor molecules are needed to reach the critical nucleation size. Fig. 4 shows that the critical nucleation size decreases from more than tens of nanometers at small surface subcooling to only a few nanometers at large surface subcooling. At a small surface subcooling, vapor molecules in the separations between nanowires cannot form clusters larger than the critical nucleation size due to its low density as well as the high energy barrier for nucleation. The high density molecules at the top side of nanowires provide a continuous supply for the cluster formation and growth to a size larger than the critical size and thus the subsequent nucleation. As a result, condensed droplets form in mobile mode on the top side of nanowires and can jump after coalescing with each other. However, under larger surface 
subcooling, fewer molecules are needed to form clusters larger than the critical size for nucleation. Droplet nucleation occurs simultaneously on all nanowire surfaces including both the top and sides of nanowires, despite the local low-density region of vapor molecules in the separation between nanowires. With the further growth and coalescence of droplets in the separations between nanowires, the water condensate will fill in the separations, leading to the pinned droplets at large surface subcooling. The nucleation-induced droplet wetting transition from mobile mode to pinned state is the fundamental root cause for the drastic differences in dynamic behaviors of condensed droplets on nanowired hydrophobic surfaces under different surface subcooling (Supplementary Section 5).

\section{Conclusions}

In summary, we reported a nanowired hydrophobic surface with significantly enhanced condensation heat transfer over a wide range of surface subcooling. By regulating the density difference of vapor close to the condensing surface through the spatial confinement effect, such a novel nanowired hydrophobic surface mitigates droplet formation in the separations between nanowires, which leads to the mobile droplets. As a result, 100\% higher overall heat flux compared to dropwise condensation on state-of-the-art hydrophobic surfaces was realized due to the highly efficient droplet jumping. The surface subcooling range of enhanced condensation heat transfer was greatly broadened, up to $24 \mathrm{~K}$, in comparison with the previously reported enhanced condensation over a very small surface subcooling $(\Delta T<5 \mathrm{~K})$. The insights gained from this work offer new opportunities to design advanced nanostructures to achieve active control of droplet nucleation and wetting, which can be applied to a diverse range of technological applications, including self-cleaning, anti-icing, water-harvesting and heat transfer enhancement. 


\section{Acknowledgment}

The authors acknowledge the financial support of this work by the National Natural Science

Foundation of China (Grant No. 51236002), the Ningbo Science and Technology Innovation Team in China (Grant No. 2014B82004), the Natural Science Foundation of Jiangsu Province in China (Grant No. BK20160935), and the US Department of Energy's ARPA-E (Contract No. DE-AR0000580). 


\section{References}

[1] A.R. Parker, C.R. Lawrence, Nature, 414 (2001) 33-34.

[2] Y.M. Zheng, H. Bai, Z.B. Huang, X.L. Tian, F.Q. Nie, Y. Zhao, J. Zhai, L. Jiang, Nature, 463 (2010) 640-643.

[3] K.M. Wisdom, J.A. Watson, X.P. Qu, F.J. Liu, G.S. Watson, C.H. Chen, Proc. Natl. Acad. Sci. USA, 110 (2013) 7992-7997.

[4] S.C. Thickett, C. Neto, A.T. Harris, Adv. Mater., 23 (2011) 3718-3722.

[5] J. Ju, H. Bai, Y. Zheng, T. Zhao, R. Fang, L. Jiang, Nat. Commun., 3 (2012) 1247.

[6] D. Attinger, C. Frankiewicz, A.R. Betz, T.M. Schutzius, R. Ganguly, A.K. Das, C.-J. Kim, C.M. Megaridis, MRS Energy Sustain. A Rev., 1 (2014) 1-40.

[7] J. Lee, S. Mahendra, P.J.J. Alvarez, ACS Nano, 4 (2010) 3580-3590.

[8] S. Daniel, M.K. Chaudhury, J.C. Chen, Science, 291 (2001) 633-636.

[9] N. Miljkovic, D.J. Preston, R. Enright, E.N. Wang, ACS Nano, 7 (2013) 11043-11054.

[10] J. Bian, Q. Li, C. Huang, J. Li, Y. Guo, M. Zaw, R.-Q. Zhang, Nano Energy, 15 (2015) 353361.

[11] D.J. Preston, D.L. Mafra, N. Miljkovic, J. Kong, E.N. Wang, Nano Lett., 15 (2015) 29022909.

[12] R. Enright, N. Miljkovic, A. Al-Obeidi, C.V. Thompson, E.N. Wang, Langmuir, 28 (2012) 14424-14432.

[13] S. Anand, A.T. Paxson, R. Dhiman, J.D. Smith, K.K. Varanasi, ACS Nano, 6 (2012) 1012210129.

[14] R.F. Wen, Z. Lan, B.L. Peng, W. Xu, X.H. Ma, Y.Q. Cheng, J. Heat Transfer, 138 (2016) 071501.

[15] J.W. Rose, Proc. Inst. Mech. Eng. A, 216 (2002) 115-128.

[16] R. Enright, N. Miljkovic, J.L. Alvarado, K. Kim, J.W. Rose, Nanoscale Microscale Thermophys. Eng., 18 (2014) 223-250.

[17] K. Rykaczewski, A.T. Paxson, M. Staymates, M.L. Walker, X.D. Sun, S. Anand, S. Srinivasan, G.H. McKinley, J. Chinn, J.H.J. Scott, K.K. Varanasi, Sci. Rep., 4 (2014) 4158.

[18] Z. Lan, X.H. Ma, S.F. Wang, M.Z. Wang, X.N. Li, Chem. Eng. J., 156 (2010) 546-552.

[19] T. Liu, C. Kim, Science, 346 (2014) 1096-1100.

[20] K.H. Chu, R. Xiao, E.N. Wang, Nat. Mater., 9 (2010) 413-417.

[21] Y. Cho, T.S. Shim, S. Yang, Adv. Mater., 28 (2016) 1433-1439.

[22] N.A. Malvadkar, M.J. Hancock, K. Sekeroglu, W.J. Dressick, M.C. Demirel, Nat. Mater., 9 (2010) 1023-1028.

[23] J.B. Boreyko, C.H. Chen, Phys. Rev. Lett., 103 (2009) 184501.

[24] C.W. Lo, C.C. Wang, M.C. Lu, ACS Appl. Mater. Interfaces, 6 (2014) 14353-14359. 
[25] C. Lv, P. Hao, X. Zhang, F. He, ACS Nano, 9 (2015) 12311-12319.

[26] N. Miljkovic, R. Enright, Y. Nam, K. Lopez, N. Dou, J. Sack, E.N. Wang, Nano Lett., 13 (2013) 179-187.

[27] N. Miljkovic, R. Enright, E.N. Wang, ACS Nano, 6 (2012) 1776-1785.

[28] X.M. Chen, J. Wu, R.Y. Ma, M. Hua, N. Koratkar, S.H. Yao, Z.K. Wang, Adv. Funct. Mater., 21 (2011) 4617-4623.

[29] C.C. Liu, J. Ju, Y.M. Zheng, L. Jiang, ACS Nano, 8 (2014) 1321-1329.

[30] R. Enright, N. Miljkovic, J. Sprittles, K. Nolan, R. Mitchell, E.N. Wang, ACS Nano, 8 (2014) 10352-10362.

[31] K. Zhang, F. Liu, A.J. Williams, X. Qu, J.J. Feng, C.H. Chen, Phys. Rev. Lett., 115 (2015) 074502 .

[32] T.M. Schutzius, S. Jung, T. Maitra, G. Graeber, M. Kohme, D. Poulikakos, Nature, 527 (2015) 82-85.

[33] S. Jung, M.K. Tiwari, N.V. Doan, D. Poulikakos, Nat. Commun., 3 (2012) 615.

[34] S.-B. Jeon, D. Kim, G.-W. Yoon, J.-B. Yoon, Y.-K. Choi, Nano Energy, 12 (2015) 636-645.

[35] N. Miljkovic, D.J. Preston, R. Enright, E.N. Wang, Nat. Commun., 4 (2013) 2517.

[36] K.K. Varanasi, M. Hsu, N. Bhate, W.S. Yang, T. Deng, Appl. Phys. Lett., 95 (2009) 094101.

[37] Y.M. Hou, M. Yu, X.M. Chen, Z.K. Wang, S.H. Yao, ACS Nano, 9 (2015) 71-81.

[38] M.D. Porter, T.B. Bright, D.L. Allara, C.E.D. Chidsey, J. Am. Chem. Soc., 109 (1987) 3559-3568.

[39] R.F. Wen, Z. Lan, B.L. Peng, W. Xu, H.B. Ma, Appl. Therm. Eng., 88 (2015) 265-273.

[40] A. Dillmann, G.E.A. Meier, J. Chem. Phys., 94 (1991) 3872-3884.

[41] D. Reguera, H. Reiss, Phys. Rev. Lett., 93 (2004) 165701.

[42] J. Rumaa, Chem. Eng. J., 16 (1978) 171-176. 

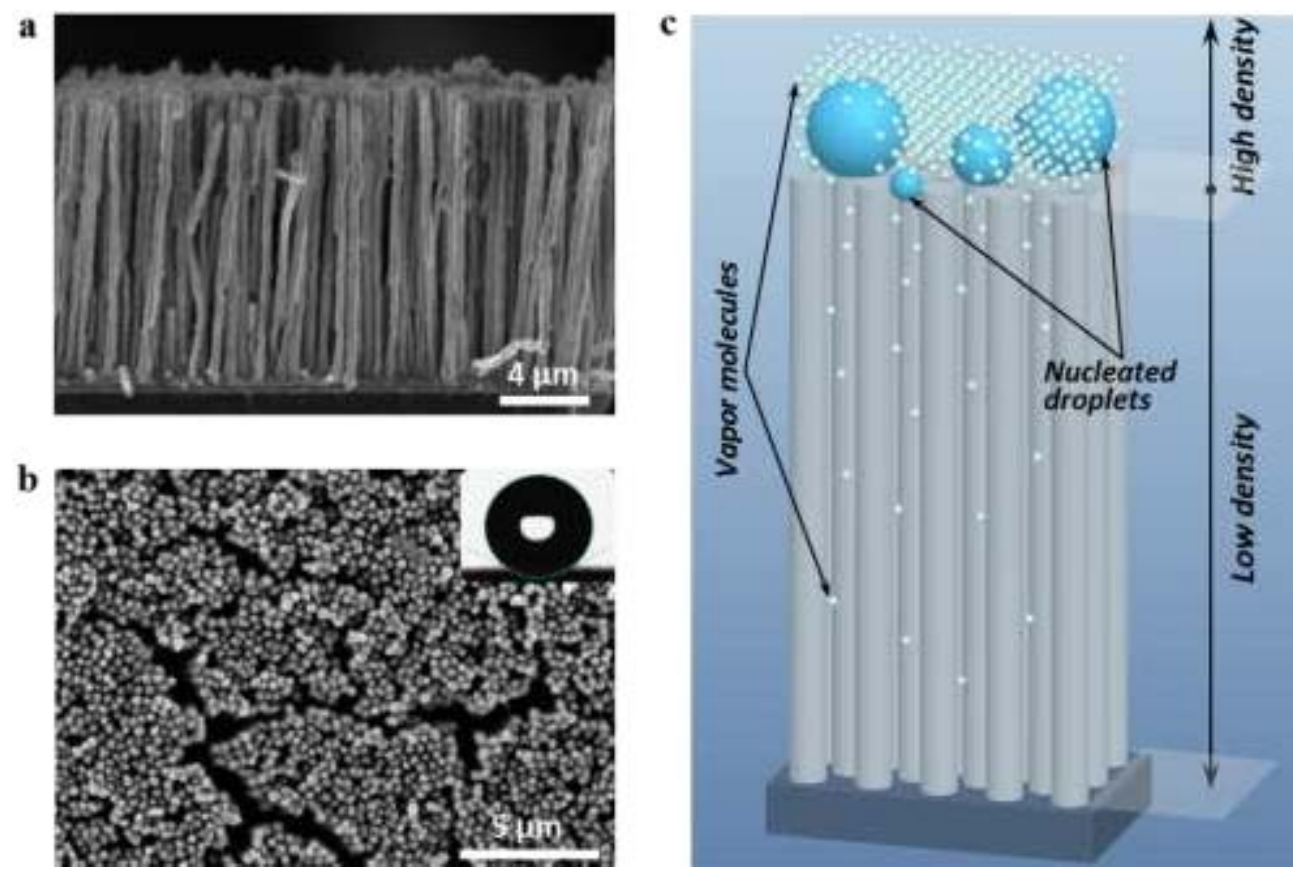

Fig. 1 Hydrophobic copper nanowires. (a-b) Scanning electron microscopy (SEM) images of the hydrophobic copper nanowires: (a) cross-sectional and (b) top view. Characteristic sizes: diameter $d=$ 200-240 nm, height $h=20 \mu \mathrm{m}$, separation $l=100-140 \mathrm{~nm}$, and center-to-center spacing $w=300-380 \mathrm{~nm}$. Inset: a $5 \mu \mathrm{L}$ droplet of water sitting on the nanowired hydrophobic surface with an apparent contact angle of $139.2 \pm 3.5^{\circ}$. (c) Schematic illustrating spatial confinement effect that prohibits droplet formation in the separations between nanowires due to the reduced permeation of vapor molecules into the separations. 


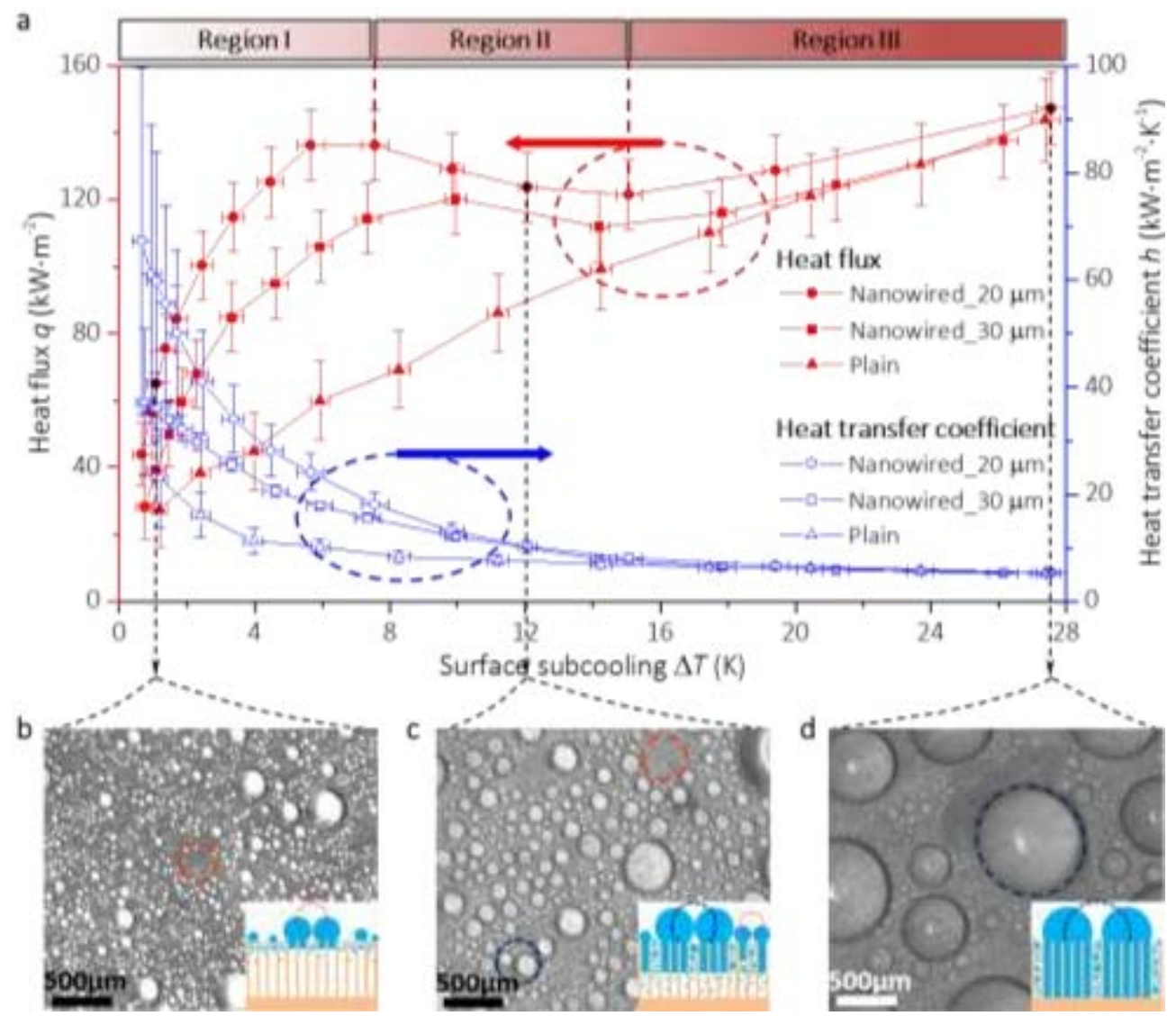

Fig. 2 Enhanced condensation heat transfer on hydrophobic nanowired surfaces. (a) Heat transfer performance (left: heat flux $q$, right: heat transfer coefficient $h$ ) as a function of surface subcooling $\Delta T$ on nanowired hydrophobic surfaces with $20 \mu \mathrm{m}$ long and $30 \mu \mathrm{m}$ long nanowires in comparison with the plain hydrophobic surface. Heat transfer enhancement can be divided into three regimes (condensation modes) according to droplet behaviors under different surface subcooling: (b) jumping condensation; (c) mixing condensation; (d) dropwise condensation. Red dotted circles highlight fresh surface areas right after droplet jumping, while deep blue dotted circles highlight areas of the surface with droplet just after coalescence and stay on surface. Scale bars: $500 \mu \mathrm{m}$. The insets in (b-d) illustrate the droplet wetting states on nanowired hydrophobic surfaces. 

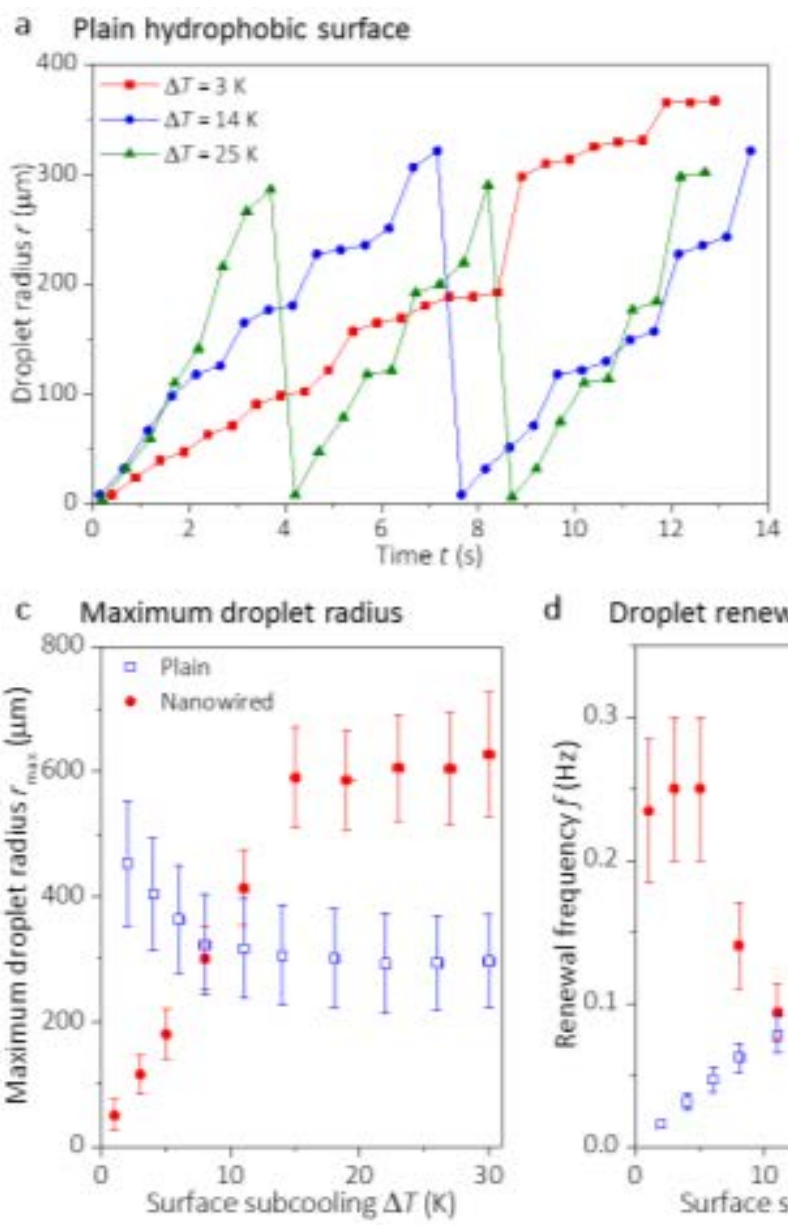

b Nanowired hydrophobic surface

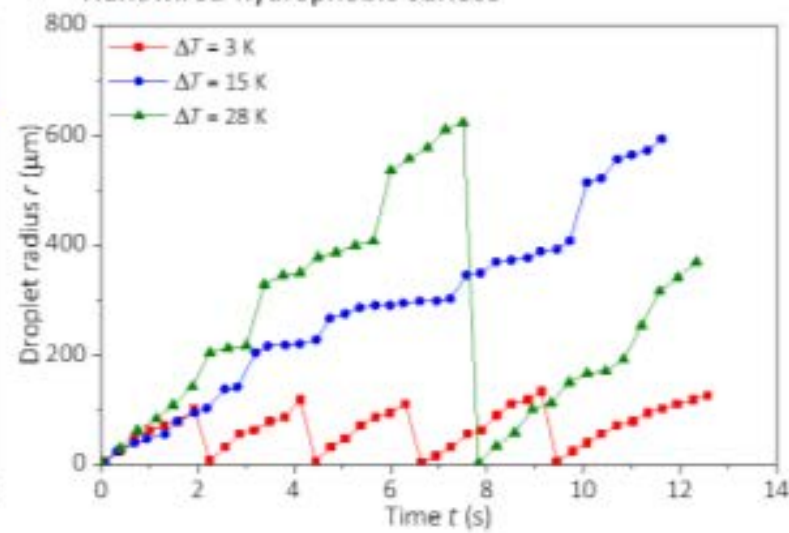

d Droplet renewal frequency

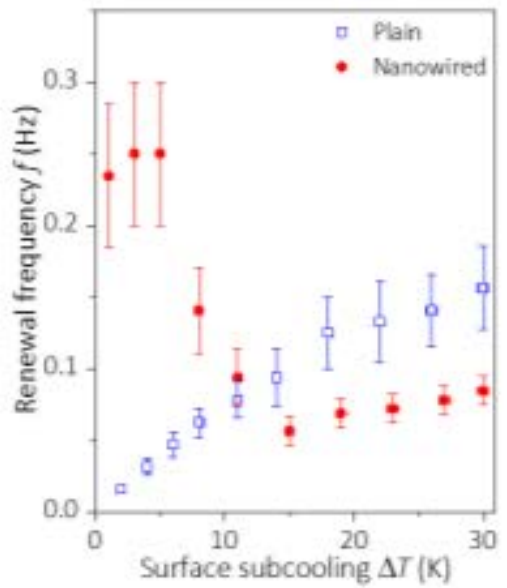

e Mean volume flux of condensate

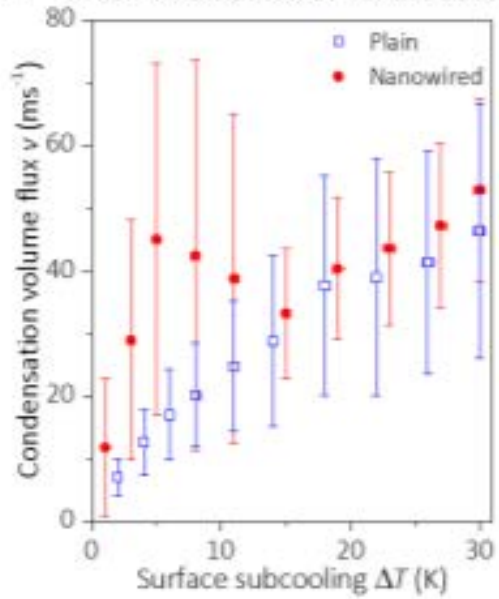

Fig. 3 Droplet growth and departure characteristics. (a-b) Typical growth history of a droplet from the initial formation to removal (shown as droplet radius reaches its maximum before the formation of another one) on plain hydrophobic surface and nanowired hydrophobic surface (20 $\mu \mathrm{m}$ long). The maximum radius (c) and renewal frequency (d) of condensed droplets as a function of surface subcooling. (e) The mean volume flux of condensate on both plain and nanowired hydrophobic surfaces at various surface subcooling. 


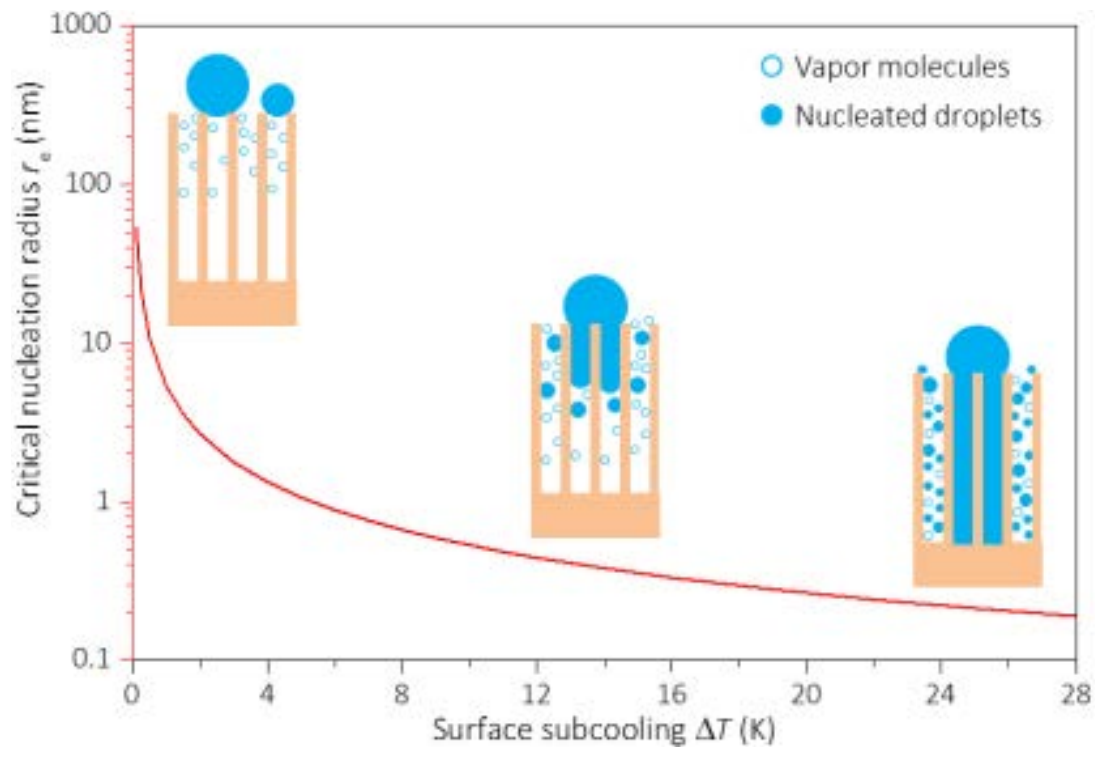

Fig. 4 Droplet wetting transition controlled by initial nucleation. The critical size for initial droplet nucleation decrease rapidly with the increase of surface subcooling. 


\section{Graphical Abstract}
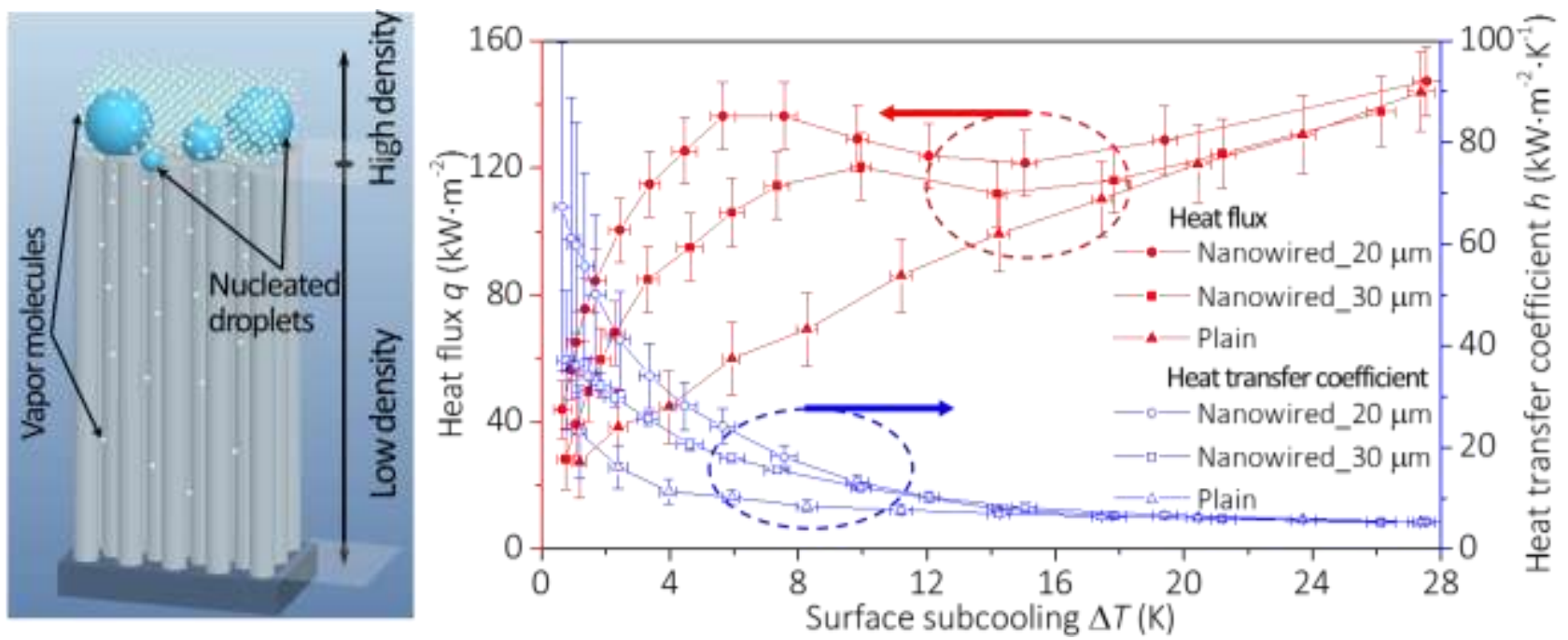

Rapid droplet removal by regulating surface topology and wettability has been exploited in nature and it is of great importance for a broad range of industrial applications. Recently there have been tremendous efforts in developing nanostructured surfaces for wettability control and enhancing phase-change heat transfer. However, the tendency of condensed droplets to form as pinned state rather than mobile mode on the nanostructured surfaces is likely to limit the applicability of such functionalized surfaces for condensation heat transfer enhancement. Here, we demonstrate enhanced condensation heat transfer on nanowired hydrophobic copper surfaces where molecular permeation of water vapor into the separations between nanowires is greatly decreased, rendering spatial control on droplet nucleation and wetting dynamics. We show experimentally and theoretically that this novel strategy allows to achieve a $100 \%$ higher overall heat flux over a broadened subcooling range, up to $24 \mathrm{~K}$, due to highly efficient droplet jumping compared to the state-of-the-art plain hydrophobic surfaces. These findings reveal that the droplet behaviors and condensation modes can be regulated by spatially controlling the nucleation events on the nanostructured surfaces, which paves the way for the design of nanostructured surfaces for enhanced phase-change heat transfer. 